\title{
Peter Derleder Die Mündigkeit der Unmündigen Kindeswohl - Kinderrechte - Kinderpflichten
}

Probleme der Kindschaftsrechtsreform werden gleichermaßen realgeschichtlich und normativ behandelt. Thematisiert wird die Veränderung der Lebensweise von Kindern und Jugendlichen, vor allem im Blick auf die Lockerung der Kohärenz der Familie. Die Frage der normativen Bestimmung von Kindesrechten steht selber im Zusammenhang mit Gruppeninteressen, vor allem von Frauen und Männern.

Die Red.

\section{Einleitung}

Eine Kindschaftsrechtsreform' steht bevor. Sie will den Eltern cincs nichtehclichen Kindes das gemeinsame Sorgerecht verschaffen, die gemeinsame Sorge der ehelich verbundenen Eltern bei Trennung und Scheidung fördern, die Betreuungsrechte der Väter nichrehelicher Kinder ausweiten, dic Frauen vom Amtsvormund für das nichteheliche Kind befreicn und dieses im Erbrecht wie das eheliche Kind behandeln. Die Intervention des Gesetzgebers in Ehe und Familie vollzieht nicht einfach Wertungen nach, die sich in der Gesellschaft bereits durchgesetzt haben, sondern beschleunigt cinen Prozeß in Richtung auf neue familiale Unübersichtlichkeit. Die Gesetzgebungsakteure berufen sich permanent auf das Kindeswohl, auch für die untcrschiedlichsten Lösungen. In den nächsten Jahren werden die Widersprüche der Reform zu verarbeiten sein, dic gern von Kindesrechten spricht, wo es um Frauenrcchte geht (. B. bei der Abschaffung des Amtsvormundes für die Feststellung der Vaterschaft und die Schaffung eines Unterhaltstitels), bci der Frauenrechte leicht Männerrechre werden (hinsichtlich der Sorge für das nichteheliche Kind) und Elternrechte womöglich Anwaltsrechte werden (durch den Anwalt des Kindes). Vor der Verstrickung in dicse Paradoxien erscheint eine grundsätzlichere Orientierung darüber notwendig, wie der Diskurs über Kindheit familiensoziologisch und rechrlich wcitcrzuführen ist.

Die Kindheit war die Entdeckung des 18. Jahrhunderts², die Familie die des 19.', die Proklamation eines Jahrhunderts des Kindes galt dem unseren. ${ }^{4}$ Nach den uns ge-

1 Siehe den Entwurf des Gesetzes zur Reform des Kindschaftsrechts, BT-Drucks. $13 / 4899$ Y. 13.6. 1996, dessen Verabschiedung in dieser Legislaturperiode nach Abschluß der Beratungen des Rechisausschusses trotz aller Einwande gesichert erscheint.

2 Pars pro toto: Jean-Jacques Rousseau, Emile oder Über die Erziehung, 1762 in Paris publizıert und zunachst gerichthich sowie durch einen Hirtenbrief des Erzbischofs von Paris geachtet. Seir dem i $\$$. Jahrhundert ist immer wieder von der "Entdeckung ${ }^{2}$ der Kindheit die Rede. $\times$ Christoph Kolumbus hat nur Amerika entdeckt, ich habe das Kınd entdeckt $\alpha$ : In dieser Selbstberuhmung Victor Hugos steckt schon die allgememe Einsicht m die Bedeutung der Kindhet fur die gesellschaftliche Moglichkeit menschlichen Glucks, wie sie die Philosophe der Aufklarung postulierte.

3 Der Begriff der Familie setzt sich erst um Laufe des 19. Jahrhunderts durch. Besonders dazu beigetragen hat dazu Wilhelm Heınrich Richl, Die Familie, Stuttgart 1859, dessen „Heiligtuma Familie dic Antwort auf die gesellschaftlichen Entwurfe der Sozialisten war.

4 Ellen Keys Prophezciung fur das zwanzigste Jahrhundert war zugleich gegen die antirousseauistischen Stromungen des 19. Jahrhunderts gerichtet, die den Kindem eine verderbte Natur und schlechte Veranla- 
wohnten wissenschaftlichen Maßstäben unter Einbezichung von Psychologie und Psychoanalyse wissen wir wenig über die Kindheiten früherer Jahrhunderte. Der Arzt Heroard, um 1700 Betreuer der Kindheit Ludwigs XIII, und sein Tagcbuch sind einzigartige Zeugen einer feudalaristokratischen Erziehung, in der das Kind wie ein kleiner Erwachsener aufwuchs, wic es ab dem 7. Geburtstag in die Händc männlicher Erzieher geriet und wie statt dem Spiel mit Puppen der Umgang mit Waffen auf den Plan gesetzt wurde.' Das Kind wurdc von Anfang an auf seine Rolle in Erwachsenenleben vorbercitet. Ariès hat in sciner Geschichte der Kindheit ${ }^{6}$, bei der cr zu einem erheblichen 'l'cil auf die Aussagekraft von Gemälden vertraute und vertrauen mußte, diesen Gedanken vom Kind als kleinem Erwachsenen verallgemeinert. Dies kann nur für eine Familien- und Gesellschaftsstruktur richtig sein, in der die Übernahme der gesellschaftlichen Stellung, der Berufsrolle und der sozialen Kompetenzen der Eltern erwartet wurde und das eigene Erwachsenenleben des Kindes zu prägen versprach. Introspektionen in die kindliche Seele waren belanglos, so daß auch noch Piagct ${ }^{7}$, als er die Entwicklungsstufen des moralischen Urteils bcim Kinde zu erforschen begann, die Kommunikation mit seinem eigenen Kind suchen konnte, als ob es noch nie ein liebevoll-neugieriges Vater-Kind-Verhältnis gegeben habc. Sclbst die großen Autobiographien vergangener Jahrhunderte enthalten von heute aus gesehen wenig Substantielles zu der frühen Kindheit.

Der vielleicht großartigste literarische Text autobiographischer Kindheit entsteht dann erst zu Beginn dieses Jahrhunderts, geschrieben über eine Kindheit der yoer Jahre des vorigen, die wirtschaftshistorisch mit dem Niedergang handwerklicher gegenüber industrieller Produktionsweise verbunden war. Er wendet sich den unteren Volksschichten zu, cntwirft aber nicht nur ein verständnisvoll objektivierendes Panorama im Stile Zolas, sondern rekonstruiert eine Subjcktivität des Kindes, dessen eigenständige Beobachtungen, Wandlungen und Haltungen auch in den Särzen des 45jährigen Autors noch durchschimmern:

"In dem halbdunklen engen Zimmer liegı auf dem Fußboden am Fenster mein Vater, er ist weiß gekleidet und ungewohnlich lang. Die Zehen seiner bloßen I-̈̈ße sind sonderbar gespreizt, und auch die Finger an seinen gütigen Händen, die still auf der Brust ruhen, sind gekrümmı. Schwärzliche Scheiben von Kupfermunzen verschließen die heiteren Augen, sein gutes Gesicht ist dunkel, und seine unschön gebleckren Zahne erschrecken mich."

Es handelt sich um dic Kindheit Alexejs, der sich später Maxim Gorki nannte, dessen crinnerte Jahre mit dem Cholcratod des Vaters beginnen und dessen . Mutter ihn deswegen in den großväterlichen Färberhaushalt nach Nishnij Nowgorod geben mußte. Der kindliche Blick, den die Länge des aufgebahrten Vaterkörpers erstaunt, nimmt die Welt der großelterlichen Großfamilie mit dem angestellten Meister, den Arbeitern und den Mietern, den vorzeitig das Erbe verlangenden Söhnen, den Prügeln für Frau und Kinder, dem Nebeneinander von archaischer Religiositär, barbarischer Auseinandersetzung und kultureller Tradition mit Märchen und Gedichten als schwer begreiflich wahr, einen Kosmos, der nur Erwachsenc zusammenhält, die für ihre Zugehörigkeit den norwendigen ökonomischen Beitrag zu leisten in der Lage sind. Der erblindetc Meister wird demzufolge ebenso zum Bcttler wic am Endc

gungen attestierten und deren Elımınierung mit Disziplinıerungen und Strafen predigten. Zu den Formen der Bestrafung, die bis zum Besuch der Galgen mit den gehenkten Verbrechern reichte, s. etwa Robertson, un: Lloyd de Mause (Hrsg.), Horr thr die Kinder weinen?, 1977, S. $565 \mathrm{ff}$.

5 Die Etappen spiegelten sich in der Kleidung. Am 6. Juni 1608 , Ludwig ist 7 Jahre und 8 Monate alt, ist er erstmals in Wams und Kniehosen gekletdet und nimmt Mantel und Degen.

6 Philippe Ariès, Geschıchte der Kındheit, Munchen 1975.

7 Jean Piaget, Das moralische Urte! beim Kinde, Neudruck Frankfurt 1973.

8 Maxim Gorkı, Mene Kindhett; der 1868 geborene Autor schrieb diesen Roman ab 19ıo (Publikatıon 1914). 
der Großvater selbst, der zuvor die als Färber ohnehin wenig geeigncten und schon

gar nicht für die Umstellung auf die Industrie gerüsteten Söhne ausgezahlt und damit seinen wirtschaftlichen Nicdergang cingeleitet hat. In der Industrievorstadt, in die der Junge zcitweilig mit Mutter und Sticfvater zieht, integriert er sich in eine Jugendbande, die von Lumpensammeln und von Diebstählen lebt. Der Tod der Mutter nach einer Schwangerschaft und dic ökonomische und soziale Bankrotterklärung des Großvaters beschließen eine Kindheit, die perspcktivlos unter fremde Menschen ${ }^{9}$ führt.

Diese untergegangene Familien- und Arbeitswelt stand im Zeichen eines ökonomischen Austauschs, dessen Solidaritätsreserven für Krankheit, Alter und Tod sich als begrenzt erwiesen und der sich mit der Zurückdrängung des alten Handwerks gänzlich überlebte. Die humanen Familiennormen, dic vor allem von den Frauen gelebt wurden, konnten sich gegen dic von den Männern teilweise grausam vertretene ökonomische Realität niche mehr durchsetzen. Der Enkel lernte von vornhercin nichts mehr vom Beruf des Großvaters und versuchte, sich über den Zugang zu den AuBenscitern cine Weltorienticrung zu verschaffen. Es wird für unscre Betrachtungen zu den Rechten und Pflichten der Kinder am Ende des 20. Jahrhunderts die leitende Fragestellung scin müssen, ob sich bei der Umwälzung von der Industriegesellschaft zur Informatiunsgesellschaft, von der Moderne zur Postmoderne einc vergleichbare Orientierungslosigkeit für die nachwachsende Generation und ihre Kindheit ergibr.

\section{Die Würde des Kindes und das Kindeswohl}

Die Deklaration der Menschenrechte in der Französischen Revolution bezieht sich noch nicht explizit auf die Rechte des Kindes. Indem aber mit der Ablösung des Feudalismus, der Herausbildung der bürgerlichen Gesellschaft und der Fntwicklung des Industrialismus der gesellschaftliche Fortschritt institutionalisicrt erscheint und die Emanzipation des Individuums vorangetrieben wird, werden die Kinder zu Trägern einer besseren zukünftigen Gesellschaft. Die großen Pädagogen, Comenius, Pestalozzi, Schleiermacher, Fröbcl, Ellen Key und Maria Montessori verlangen Respekt vor dem Kind als verletzlichem Wesen, dem die Gesellschaft das Beste schuldet, das sie geben kann. ${ }^{10}$ Bei aller Ungleichzeitigkeit der gesellschaftlichen Entwicklungen nach Zeit und Raum sctzte sich dieser Gedanke der fundamentalen Verbindlichkeit gegenüber den Kindern immer mehr durch, die die Erreichung des Reiches der Freiheit verbürgen soll. Die UN verabschiedeten 1959 eine Deklaration zu den Rechten des Kindes, die durch die UNO-Kinderkonvention von 1989 "eine verbindlichere Rechtsgrundlage erhielt.

Im Grundgesetz ist vom Kindeswohl nicht ausdrücklich die Redc. Aber schon früh hat das Bundesverfassungsgericht anerkannt, daß das Kind mein Wesen mit eigener Menschenwürde und einem eigenen Recht auf Entfaltung sciner Persönlichkcit im Sinne der Art. I Abs. I und 2 Abs. I GG ist «. ${ }^{12}$ Bei der Interpretation des Art. 6 GG, dessen Abs. I den besonderen Schutz des Staates für Ehe und Familie voranstellt und

9 Maxim Gorkı, Unter fremden Menschen, 1917. Siehe ferner die Bıographie von Geir Kjetsaa, Maxim Gorkı, 1996.

10 Was in den 7oer Jahren dieses Jahrhunderts sveder aufgenommen wurde; siehe dazu besonders pointiert Hans-Joachim Gamm, Vorgange 42 (1979), S. 67 ff.

It In Kraft getreten in Deutschland am 5. 4. 1992, BT-Drucks, 12/4168.

12 BVerfGE 24, 119, 144 . 
dessen Abs. 2 die Pflege und Erziehung der Kinder als das natürliche Recht der Eltern und die ihnen zuvörderst obliegende Pflicht bezeichnet, wird das Kindeswohl jedoch von Jahrzehnt zu Jahrzehnt mehr zum entscheidenden verfassungsrechtlichen Prinzip aufgewertet. ${ }^{13}$ Das Elternrecht wird zunchmend nur noch als Elternpflicht und -verantwortung verstanden. ${ }^{14}$ Der mit dem Gesetz zur Neuregelung der elterlichen Sorge's 1980 vollzogene Begriffswechsel von der elterlichen Gewalt zur elterlichen Sorge bezieht sich explizit auf das leibliche, geistige und seclische Wohl des Kindes ${ }^{16}$ und sollte den umfassenden Pflichtcharakter der elterlichen Rechtsstellung betonen. ${ }^{17}$ Auch wenn die Kritik an der Vagheit des Begriffs "Kindeswohl « nie ganz verstummt ist ${ }^{18}$, hat sein Sicgeszug zu einer erheblichen Verfeinerung der sozialwissenschaftlichen Erkenntnisse über die Kindheit und einer tiefergehenden Rezcption durch die Rechtswissenschaft'9 sowie zu einer erklärten Umsetzungsbereitschaft der Rechtspraxis geführt. Letztere schließst eine sachverständige Konfliktaufarbeitung ein, die jedoch in den neunziger Jahren in Routinen zu erstarren droht. ${ }^{20}$ Der allzu cuphorische rhetorische Umgang mit dem Kindeswohl ist rechtzeitig dahin gedämpft worden, daß nur noch die am wenigsten schädliche Alternative für das Kind realisiert werden soll. ${ }^{21}$ Das Kindeswohl steht ideologisch höher als der Schutz der Institution Ehe, wie an der Entscheidung des Bundesverfassungsgerichts zum gemeinsamen Sorgerecht nichtehelicher Partner besonders deutlich geworden ist. ${ }^{22}$ Die Grundrechte des Kindes und seine Grundrechtsmündigkeit sind zwar nicht in allen Dimensionen geklärt, insbesondere auch nicht in verfahrensrechtlicher Hinsicht, wo der familienfremde Anwalt des Kindes auf der Tagesordnung des Gesetzgebers steht. ${ }^{33}$ Klar ist aber, daß Rechte der Eltern in der gegenwärtigen Konfliktentscheidungspraxis nicht mehr als Rechre gegenüber dem Kind, sondern allenfalls als Rechte gegenüber dem Ehegatten oder nichtehelichen Partner oder als Abwehrrechte gegenüber dem Staat postuliert werden.

Damit ist der Abschied vom sozialökonomischen Modell des familiären Generationenaustausches ${ }^{24}$ perfekt. In diesem Modell, das in der Landwirtschaft noch lange Bestand hatte, sorgt die elterliche Generation für das Aufwachsen, die Erziehung, dic Ausbildung und die Berufsintegration, während die Nachfolgegeneration dic Risiken von Krankheit und Unfall sowie die Altersbedürfnisse der Vorgeneration abdeckt, wobei - abgesehen von strafrechtlichen Sanktionen - Unterhaltsansprüche, Eigentum und Erbrecht die Einhaltung der gegenseitigen Verpflichtungen gewährleisten. Die Entwicklung der Industriegesellschaft und die Herausbildung eines freien Arbeitsmarktes für die Berufsausübung in Industrie und Dienstleistungswirtschaft haben dafür gesorgt, daß immer mehr Aulgaben in Ausbildung und Erziehung vom Staat übernommen werden mußten, daß der Staat inzwischen massiv sogar die reine Kinderbetreuung und -alimentation subventionicrt und daß umgekehrt der familiäre durch einen kollektiven Generationenvertrag zur sozialen Sicherung insbesondere

13 Siehe etwa BVerfG NJW $1993,2671$.

14 Siehe dazu insb. BVerfGE 56,363 , wo der Pflichtcharakter wals wesensbestimmender Bestandteile des Elternrechts verstanden wird.

is SorgeRG vom 18.7. 1979, BT-Drucks. $8 / 2788$

16 Siche $\$ 1666$ Abs. 1 Satz 1 BGB.

17 Siche die Begrundung des Rechtsausschusses des Bundestags, BT-Drucks. $8 / 2788$ S. $36,43$.

18 Siehe insb. Mnookin FamRz 1975, 1; stehe auch Derleder FuR 1994, 146.

19 Siche etwa insb. Coester, Das Kindeswohl als Rechtsbegriff, $198_{3}$; Hinz, Kindesschutz als Rechesschutz. und elterliches Sorgerecht, 1976; Zenz., Kindermı Shandlung und Kinderrechte, 1979.

20 Siehe Derleder (Fn. 18).

$21 \mathrm{Im}$ Anschluß an Goldstein/Freud/Solntt, Jenserts des Kindeswohls, 1974, stehe nur MunchKomm-BGBRichter, 3. Aufl., $\$ 1666$ Rdnr. 24.

22 BVerfG NJW 1991, 1944.

23 Siehe dazu die grundlegende Monographie von Salgo, Der Anwalt des Kındes, 1993.

${ }_{24}$ Siehe dazu besonders posntert Hesnsohn/Knieper, Theorie des Familienrechts, 1977. 
für Krankheit und Alter ersetzt wurde. Das Gegenseitigkeitsverhältnis der Generationen hat sich in der Familie mehr und mehr in ein einseitiges, in ein donatorisches Vcrhältnis umgewandelt, wenn man dic ökonomischen Vorgänge betrachtet.

Die Postmoderne ist nun aber dadurch geprägt, daß nicht nur die Familien die ihnen verbliebenen und neu zugewachsenen Funktionen weithin mehr schlecht als recht wahrnehmen, sondern auch der Staat in Schule und Hochschule wie hinsichtlich der Leistungsfähigkeit der sozialen Sichcrungssysteme überfordert erscheint. Eine Reaktivierung der Solidarfunktionen kleinerer Gemeinschaften, wie sie Teilen der kommunitaristischen Bewegung vorschwebt ${ }^{25}$, oder eine Rückverlagcrung auf die Familien ist wcithin nicht möglich, da weder die Familie noch eine lokalc Gemeinschaft die Ausbildung und flexible Fortbildung für das hohe Qualifikationsniveau ciner Industrie- und Informationsgcsellschaft nmit Wcltniveau « lcisten können, wo dies doch dem Experten, insbesondere Lehrcrn und Professoren, nur unzulänglich gelingt. Ob größere finanzielle Enge oder Not den Familien mehr solidarische Kraft geben können, ist mit Skepsis zu beurtcilen.

Machen wir in dem Licht dieser Entwicklungen eine Momentaufnahme zum Kindeswohl und seiner Verfassung. Kinder werden in Europa heute nur noch in geringem Umfang mit biologischer Zwangsläufigkcit geborcn. Die Empfängnisverhütungsmethoden erlauben es weitgehend, sich auf Wunschkinder vorzubereiten. Die großen Debatten um die Zulässigkeitsvoraussetzungen für die Schwangerschaftsunterbrechung drehen sich ebenfalls um die Befugnis der Frauen, sich nach sozialen Kriterien für oder gegen ein Kind zu entscheiden. Aber auch das Leben mit Wunschkindern ist hart. Familien mit Kindern sind in vielfältiger Weise eingeschränkt. Für die Eltern bedeutet ein Kind zunächst Konsumverzicht. Auf dem Wohnungsmarkt und dem Arbeitsmarkt werden Familien mit Kindern nachhaltig und systematisch benachteiligt. Der öffentliche Raum ist vor allcm aufgrund der Vorherrschaft des Autos weitgehend kinderfeindlich. Je mehr vom Kindeswohl die Rede ist, desto kinderfeindlicher ist die Umwelt. Eltern haben all dies abzuarbeiten und werden dabei einer allgemeinen staatlichen Supcrvision unterstellt.

Bei der Ausübung scines Wächteramts behandelt der Staat die unterschiedlichen Kindergruppen jedoch in sehr differenzierter Weise, wenn man einmal von der Kindergeldzahlung absieht. Besonders sorgfältig geht er mit den sog. Scheidungswaisen um, d. h. denjenigen Kindern, die von Trcnnung und Scheidung der Eltern betroffen sind. Ihre ökonomischen, sozialen und psychischen Bedingungen werden dann erkundet, durch Jugendamtsmitarbeiter und Sachverständige, bis hin zur sog. Anhörung von Kleinkindern durch den Familienrichter. ${ }^{26}$ Diese Untersuchung orienticrt sich nicht mehr an dem Trennungsverschulden, das nach altem Recht noch eine Rolle spielte. Die damaligc Erwartung an die Frau, eine unglückliche Ehe um der Kinder willen fortzuführen, ist heute weitgehend überholt. Nach der Aufgabe des Verschuldensprinzips sind die von Trennung und Scheidung betroffenen Kinder im Mittelpunkt der juristischen Erörterungen geblieben und auch jetzt hauptsächliche Adressaten der Kindschaftsrechtsreform. Ihr Schicksal ist stets mit dem der Kinder verglichen worden, die mit Vater und Mutter aufwachsen, mit teilweise dramatischer Ưberpointierung der Kausalität der Scheidung für ein späteres schweres Lebensschicksal, nicht aber im Vergleich mit einem Heranwachsen unter ständigen unfriedlichen Auseinandersetzungen zwischen den Eltern.

Erstmals drohen die Scheidungskinder ausgercchnet mit der Kindschaftsrechtsre-

25 Siehe insb. Amstai Etzioni, Die Entdeckung des Gemeinwesens, 1995.

26 Nach $\$$ sob Abs. 1 FGG genugt es, wenn sich das Gericht von dem Kind einen unmittelbaren Eindruck verschaffen kann. Auf den Diskurs mit dem Kind kommt es dabei nicht an. 
form aber auch an staatlichem Interesse zu verlieren, da bei der Scheidung die Sorgerechtsregelung nicht mehr obligatorisch mit dem Scheidungsausspruch verbunden werden soll. Die Verantwortung für die Scheidungsfolgen wird insoweit staatlicher Prüfung entzogen und den Eltern überantwortet, die vielfach mit rhetorischen Gemeinsamkeitsbekundungen hinsichtlich der Kindererziehung sich und andere über ihre tiefgreifenden Konflikte hinwegtäuschen. Dic Kinder, die von vornherein in einem Haushalt mit einem Alleinerzieher oder einer Alleinerzieherin aufwachsen, nennt niemand auch nur im übertragenen Sinn Waisen, cbensowenig die Heimkinder oder die Pflegekinder, deren leibliche Eltern von vornhercin überfordert waren. Bei dem Impcrativ, die Kinder aus den Heimen zu holen, fehlte oft die Erkenntnis, daß es konkret die schlechten Bedingungen der meisten Heimc waren, die einer zufriedenstellenden Sozialisation entgegenstanden, und daß diese Bedingungen hinreichend verbessert wcrden könncn, so daß die Erziehung in Pflegestellen nicht in jedem Fall vorzuzichen ist. Vor allem leiden die Pflegekinder nach wic vor unter dem unklaren Rechtsstatus der Pflegeelternschaft, der zu einer Vielzahl von Konflikten der leiblichen Eltern und der Pflegeeltern geführt hat. Das Paradox einer Erwartung an die Pflegeeltern, den Kindern möglichst viel Zuwendung zu geben, aber richt so viel, daß sie sich seelisch dauerhaft auf die Pflcgeeltern ausrichten, läßt gradlinige und einfache Sozialbeziehungen zwischen Pflegeeltern und -kindern kaum zu. Am Ende der Aufmerksamkeitsskala stehen schließlich die unbegleiteten ausländischen Kinder, wie wir sie in einem unerklärten Ein wanderungsland antrcffen und die dic Behörden zwischen ausländerrechtlicher Abwchrhaltung und familienrechtlicher Betreuungserwartung schwanken lassen.

Die staatliche Bemühung um das Kindeswohl ist also bei genauerer Betrachtung hochdifferenziert. Dies gilt auch für dic geltenden familienrechelichen Regelungen. So hoch das Kindeswohl theoretisch angesiedelt ist, so hoch ist praktisch die Interventionsschwellc für Familien, in denen dic Kinder mit den Eltern zusammenleben. Das Verbot entwürdigender Erziehungsmaßnahmen in $\$_{1} 6_{31}$ Abs. 2 BGB kommt zwar dem mit der Kindschaftsrechtsreform zu erwartenden Verbot körperlicher Züchtigung schon nahe. ${ }^{27}$ Wenn das Vormundschaftsgericht eingreift, geht es aber meist um eine langdauernde gravierende Vernachlässigung und Mißhandlung ${ }^{28}$, übcr die die soziale Umwelt nicht mehr hinwegschen kann. Erst bei Trennung und Scheidung, also Ereignissen, die keineswegs notwendig ein besonders schweres bisheriges Schicksal der Kinder indizieren, verdichtet sich dic sozialc Kontrolle des Staates.

Die Formen der Vernachlässigung verändern sich, ohne daß dies in der vormundschaftsgerichtlichen Praxis erkennbar würde. Im Schatten demonstrativer Ächtung des Prügelns bleibt etwa die Methode, Kinder, auch Kleinkinder, durch Fernsehkonsum stillzustellen, unerörtert. Neil Postmans Warnungen verhallen insoweit ungehört. ${ }^{29}$ Die gravierenden Konzentrationsstürungen nicht erst der lctzten Kindergcneration lassen Lehrer und Professoren die Hände über dem Kopf zusammenschlagen. Die Kinder werden so ganz früh zu Konsumenten in einem Ambiente des Genusses, vom gesüßten Schnuller bis zum narzißtischen Outfit. Im Zeichen eincr Apotheose des Kindeswohls hat sich vor allem für das erste Jahrzehnt der Kinder tcilweisc cine Lebenspraxis ergeben, die dringend cincr Inpflichtnahme der Eltern auf cinen kindergcrechten Zeithaushalt, auf soziale und kulturelle Gcmeinsamkeiten

$27 \mathrm{Vgl}$. etwa MuncbKomm-Hınz, $\$ 1666 \mathrm{Rdnr}$. 32, der betont, daß alle entwirdigenden Erziehungsmaßnahmen (auch nichtkorperlicher Art) untersagt sind, daß aber eine vereinzelt gebliebene Zuchtigung nicht relevant sei.

28 Siche dazu BayObLG FamRZ 1984,28

29 Nell Postman, Das Verschwinden der Kindhert, 1983. 
bedarf. Insoweit haben die Kindesgrundrechtc nicht nur ein gewaltiges Realisierungsdefizit, vielmehr ist hierfür auch die weitere Ausdifferenzierung der Kindesrechte notwendig.

Auch bei Familien, die mit ihren Kindern harmonisch und mit einer eigenen familialen Kultur zusammenleben, ergeben sich jedoch inzwischen meist Brüche in deren zweitem Lebensjahrzehnt. Oft beginnen die schwierigen Jahre von einer Woche zur anderen. Die bisherigen Verhaltensformen werden abgestreift. Das kann vom freundlichen Umgang mit den Nachbarn über plötzliche Änderungen des körperlichen oder kleidungsmäßigen Phänotyps bis zur schweren Lernstörung reichen. Eltern können es nicht verhindern, daß ihre Kinder an Parties mit Alkohol und Drogen teilnehmen, Pornovideos konsumieren, oft erst am frühen Morgen nach Hause zurückkehren und ihre Maßstäbe aus schwer faßbaren pecr groups beziehen. Der hingebungsvollen, ja oft ekstatischen Anhimmelung von Popstars begegnen die Eltern schon im Kinderzimmer. In Elternhäusern mit reichen Bildungsbeständen kann es zu der überraschenden Wendung kommen, daß die Kinder ihre gesellschaftlichen Erkenntnisse und literarischen Horizonte weitgehend nur noch der Zeitschrift "Bravo entnchmen wollen und demgemäß viel genauer über das Sexualleben von Rockbands Bescheid wissen als über die Struktur von Satzgetügen.

In Familien, die nie mit dem Jugendamt in Berührung kommen, kann der Lebensrhythmus von Eltern und Kindern so auseinanderfallen, daß die Gemeinsamkeiten immer geringer werden. Nach den Wochenenden mit Diskonächten steht der Alltag im Zeichen von Schlafmangel und Aggressivitäten. Eltern müssen langfristig vorfühlen, wann cine Stunde zur gemeinsamen Vorbereitung von Klassenarbeiten in Problemfächern zur Verfügung steht. Daß sich Kinder ungeachtet clterlicher Ratschläge auf eine gesundheitsgefährdende Nahrung, Kleidung und Freizeitgestaltung kaprizieren, ist bundesrepublikanischer Alltag. Eltern, dic megaout sind, können nicht erwarten, daß stets Bereitschaft zu rationalem Diskurs Konflikte auf eine für sie erträgliche Weise zu löscn vermag, sondern haben lediglich die Hoffnung, daß diese, von ihnen sog. Ekelphase bald vorübergeht und inzwischen keine dauerhaften Schäden eintreten oder gar die Weichen definitiv auf schulisches, berufliches oder privates Scheitern gestellt werden. Viele Eltern haben den Eindruck, daß sic einem Bündnis der Kinder mit sämtlichen Protagonisten des Konsums, der Devianz und des kulturellen Verfalls gegenüberstehen. Selbstertahrungsgruppen besonders verzweifelter Eltern versuchen, durch gegenseitige Information und Beratung Haltung zu bewahren. Meist ist nach einigen Jahren der Spuk vorüber, etwa nach cinem Auslandsjahr oder nach ersten beruflichen Phasen.

Die Gründe für die dramatischen kulturcllen und sozialen Absetzbewegungen Jugendlicher von ihren F.ltern sind heterogen. Maßgeblich ist die Grunderfahrung, daß die Eltern der Generation ihrer Kinder nicht mitgeben können, was dic Bewälrigung der Zukunft erfordert. Die Eltern haben weitgehend nicht mehr die Kompetenz, für Erfolge in Schule, Ausbildung und Beruf maßgebliche Beiträge zu liefern. Sie können keinen qualifizierten Rat zur Bcrufswall erteilen und nur in seltenen Fällen eigene Berufsqualifikationen weitergeben. Ein weirerer Grund ist der revolutionäre Wandel im Geschlechterverhältnis während der letzten Jahrzehnte, der Ehe und Partnerschaft der Eltern und ihre soziale Integration meist nicht als Muster zugrunde zu legen erlaubr. Die Jugendlichen sind zudem Adressaten sich stetig überholender Angebore für Konsum und Frcizcit, die die Heranbildung zum konsumistischen Jung-Single begünstigen. Dabei kann auch der noch im elterlichen Haushalt ("Hotel Mama a) lebende, sich aber als Single verstehende Jugendliche cinen so vollen Terminkalender haben, daß er sich sters im Streß fühlt. Neben Schule, Job, Fernsehen, Parties, vielfältigen Vcrabredungen, ausgedehnten Konsumerkundungen etc. bleibr 
kaum Zeir zu ausreichendem Schlaf. Die Stile wechseln, der Musikgeschmack erwa so stark, daß sich junge Leute über einen Altersabstand von einem Jahrzchnt hinweg kaum über gemeinsame Musikinteressen verständigen können. Natürlich kann die kulturelle Verwerfung auch durch eltcrliche Nachlässigkeit im crstcn Lebensjahrzehnt begünstigt sein. Faszinierend ist aber gerade der zu beobachtende abrupte Wechscl von der Konformitäts-zur Konfliktperiode. Eltern müssen hcute manchmal froh sein, wenn ihre treuherzige, fleißig Geige spielende Tochter nicht plötzlich das Instrument und die Abiturpläne wegwirft und mit einem verwclkenden Großvatertyp ins soziale und berufliche Nirwana aufbricht.

Es ist kcine Frage, daß noch jede Elterngeneration über Flcgeljahre und Ablösungsprozesse der Nachfolgegeneration geklagt hat. Das Tempo des gesellschaftlichen Wandels hat aber entscheidend zugenommen. Die Ablösung von den Eltern ist nicht nur psychoanalytisch notwendig, sondern auch sozial zwangsläufig. Dicse Ablösung schließt prinzipiell auch das Recht ein, zeitweilig in Sackgassen zu geraten. Verantwortungsvolic Eltern können es zwar kaum ertragen, wenn sie sehen müssen, wie ihre Kinder ganz früh Um- oder Abwege einschlagen. Die Eltcrn drogengefährdeter Kinder suchen oft nur zu sehr die Schuld bci sich. Der elterliche Einfluß ist während der kritischen Jahre außerordentlich gering geworden, wobei der Wcgfall des familiären Generationcnaustauschs die sozialökonomische Grundlage ist. Es ist daher kein Wunder, wenn zur Entschärfung der Konflikte zwischen Eltern und Kindern auch an Kinderpflichten neben den Kinderrechten gedacht wird.

Anknüpfungspunkte dafür gibt es früh. Schon kleine Kinder wollen zum familiären Miteinander beitragen, durch Rituale des Gebens und Nehmens. Die Austauschprozessc des Alltags sind das Feld, in dem auch Dreijährige bereits Anerkennung suchen. Der bcrufstätige Elternteil, der eine gevwisse Rücksichtnahme auf seinen Beruf fordert, kann dies manchmal besser vermitteln, als der auf Haushaltsdicnstleistungen beschränktc. Viclc Kinder wissen früh, daß sie etwas dazu beitragen müssen, wenn die Mutter im Beruf bestehen soll, und kümmern sich um die Minderung ihrer Doppelbelastung.

Mit der Formulierung eines Ideals allerdings, nach dem Eltern ihren Kindern gegenseitige Hilfe und Solidarität vorleben sollen, ist es aber nicht getan. In zweiten Lebensjahrzehnt haben die meisten Kindor zu Recht das Gefühl, daß ihnen Eltern das nicht mehr geben können, was für ihre Zukunft wichtig ist, so daß der Austauschprozeß grundlegend gestört ist. Das ist die Ursachc dafür, warum rechtliche Verpflichtungen der Kinder und sogar Sanktionen ins Blickfeld rücken. Machen wir demgemäß einen Streifzug durch die geltenden gesetzlichen Regelungen, die Kinderpflichten oder -obliegenheiten enthalten können. Die Kindschaftsrechtsreform hat sie nicht im Blick und bringt insoweit keinc Änderungen.

\section{Der Unterhaltsverband als Pflichtengefüge}

$x$. Nach $₫$ r 6or BGB haben Verwandte in grader Linic einander Unterhalt zu gewähren. Diese Norm ist immer noch auf den Generationenaustausch innerhalb der Familie einschließlich der Großfamilie abgestimmt, entspricht aber nicht mehr den realen Verhältnissen. Die Unterhaltspflicht bezieht sich dagegen praktisch im wesentlichen auf die Kernfamilie. Die Unterhaltspflicht der Eltern gegenüber den Kindern ist im einzelnen mit Tabellen und Richtlinien ausdifferenziert. ${ }^{30}$ 
Die umgekehrte Unterhaltspflicht der Kinder gegenüber den Eltern wird mit Rücksicht auf die kollektive Absicherung der Eltern weitgehend nicht mehr in Anspruch genommen. Soweit alt gewordene Eltern ins Heim kommen oder für sie Pflegeleistungen zu erbringen sind, die nicht sozialversicherungsrechtlich gedeckt sind, kann im Wege des Unterhaltsregresses die nachfolgende Generation zwar in Anspruch genommen werden, insbesondere nach $\$$ gr BSHG. Damit kann die nachfolgende Generation in große Schwierigkeiten geraten, wenn sie ihrerseits wieder für ihre Kinder zu sorgen hat (als sog. Sandwich-Generation). In jedem Fall wird der Unterhaltsregreß gegenüber den Kindern durch einen höheren Selbstbehalt beschränkt. ${ }^{3:}$ Bisher reicht das Sozialversicherungssystem aus, um die Unterhaltspflichten der nachfolgenden Generation auf einen rclativ kleinen Anteil der Familien zu beschränken. Ob dies im Zuge des Abbaus des Sozialstaats so bleiben wird, ist jedoch 7.u bezweifeln.

2. Der Unterhaltsanspruch der Kinder gegenüber ihren Eltern ist bis in kleinste Rechendetail reguliert. Die wenigsten Kinder verfügen über eigenes Vermögen und sind deswegen unterhaltsbedürftig. Den Stamm eines vorhandenen Vermögens muß ein minderjähriges unverheiratetes Kind nur angreifen, wenn die Eltern nicht leistungsfähig sind $(\$ 1603$ Abs. 2 Satz 2 BGB). Im übrigen ist das minderjährige unverheiratete Kind unterhaltsberechtigt, soweit die Einkünfre aus eincm etwa vorhandenen Vermögen oder anderes Einkommen nicht ausreichen, wie dies in aller Regel der Fall ist.

Die Unterhaltsbedürftigkeit eines Kindes ist zu verneinen, wenn cs seiner Erwerbsobliegenheit nicht genügt. Kinder haben jedoch bis zum Abschluß der Ausbildung praktisch keine Erwerbsobliegenheit. In cinem Einzelfall hat die Rechtsprechung einmal eine Erwerbsobliegenheit bejaht, wo ein minderjähriges Kind noch einige Zeit bis zum Beginn der Ausbildung warten mußte. ${ }^{32}$ Auch ein Student braucht im Regelfall nicht einen Teil seines Unterhalts durch eine Nebenbeschäftigung zu verdienen, um seine leistungsfähigen Eltern zu entlasten. ${ }^{33}$ Nach Abschluß einer Berufsausbildung oder deren Abbruch sind Kinder jedoch verpflichtet, eine Arbeit aufzunchmen, notfalls auch eine unterhalb der gewohnten Lebensstellung. ${ }^{3+4}$

3. Eine erhebliche Rolle für die gegenseitige Rücksichtnahme von Eltern und Kindern spielt $\$ 16 r_{2}$ BGB. Danach ist der Unterhalt grundsätzlich durch die Entrichtung einer Geldrente zu gewähren, nicht also durch Betreuungs- und Naturalleistungen (sog. Naturalunterhalt). Haben die Eltern einem unverheirateten, auch eventuell volljährigen Kind Unterhalt zu gewähren, so können sie bestimmen, in welcher Art und für welche Zeit im voraus der Unterhalt gewährt werden soll ( $\$$ I6 I 2 Abs. 2 Satz I BGB). Die meisten Eltern machen von ihrem Bestimmungsrecht, wenn das überhaupt möglich ist, in der Weise Gebrauch, daß sie Unterhalt in Natur gewähren. Das ist möglich, indem das Kind im elterlichen Hause wohnt, soweit wie möglich an den Mahlzeiten teilnimmt und auch seine Wäsche hier gewaschen wird. Auch sonst können gemeinsame Einrichtungen genut\%t werden. Der Rationalisierungseffekt des gemeinsamen Lebens in einem Haushalt bedeutet für viele Eltern eine wesentliche finanzielle Entlastung. Diese tritt auch ein, wenn ein Kind im gemeinsamen Haus eine getrennte Wohnung bewohnt. Diese ökonomisch günstige Lösung kann aber

die aber mecht ein Gewirr von weitcren Tabellen und Letslinten verhindert hat, be denen die meisten Oberlandesgerichte trotz grassierender Ide'cnarmut ihre Interprerationsherrschaft zu dokumentieren versuchen.

31 BGH NJW 1992, 1393.

32 OLG Karlsruhe FamRZ 1988, 758.

3) KG FamRZ 1982, 516.

34 BGH FamRZ, $1985,273$. 

angesprochen sind.

Ein Zusammenlcben ist andererscits bei älteren Kindern, insbesondere volljährigen, häufig mit erheblichen Konflikten belastet. Die Kinder wollen sich nicht in den elterlichen Rhythmus einfügen, sich von Elternhaus lösen und ihr Lcben selbständig führen. Demgemäß stehen sich hier das berechtigte Selbständigkeitsinteresse des Kindes und das berechtigte ökonomische Interesse der Eltern gegenüber, die bei Zahlung von Barunterhalt auf ein sehr niedriges Resteinkommen beschränkt werden können. Sind mehrere Kinder vorhanden, kann sich diese Situation noch zuspitzen. "Aus besonderen Gründen « kann das Vormundschaftsgericht auf Antrag des Kindes die Bestimmung der Eltern ändern. Dazu gibt es eine reiche Judikatur, die konkretisiert, unter welchen Umständen es insbesondere einem volljährigen Kind nich mehr zuzumuten ist, mit den Eltern zusammerzuleben. Die Rechtsprechung stellt hier vor allem darauf $a b$, ob eine riefgreifende Entfremdung zwischen Eltern und Kind festzustellen, aber teilweise auch darauf, auf wen diesc Entfremdung zurückzuführen ist. So werden erwa die "7.wangsweise unangemessene Einflußnahme auf die Lebensführung des Kindes«, die Vorenthaltung von Wohnungsschlüsseln, ein schikanöser Verhaltenskodex für die Zeiten der Anwesenheit und Abwesenheit sowie Mißtrauensbekundungen und übergroße unpersönliche Härte als wesentlich angesehen. ${ }^{35}$

In der Literatur heißt es etwa, es sei auf die Einhaltung der von den Eltcrn bestimmten Mahlzciten zu achten, wenn nicht dringende Gründe entgegenstünden, aber auch auf äußerste Regelzeiten für das morgendliche Aufstehen oder das abendliche oder nächtliche Nachhausckommen, soweit der gemcinsamc Haushalt dies vernünfrigerweise bedinge; darüber hinaus müsse das Kind auch als Volljähriger in der elterlichen Wohnung diejenigen Rücksichten nehmen, die von jedem Dritten auch erwartet werden könnten. Habe das Kind grundsätzlich andere Vorstellungen, so sei die gegenseitige Rücksichtnahme und ein beiderseitiges Entyegenkommen und Nachgeben erforderlich. Seien die Stand punkte miteinander unvereinbar, müsse der gerncinsame übereinstimmende Vorschlag beider Eltern im Zweifel auch beim Vormundschaftsgericht den Ausschlag geben. ${ }^{36}$ Voller Konsens darüber besteht allerdings nicht.

Als Gründe für die Aufkündigung des Zusammenlebens und die Zahlung von Barunterhalt werden in der Rechtsprechung Ohrfeigen und die Herabwürdigung der (fast) volljährigen Tochter durch den Vater ${ }^{37}$, fehlende Toleranz gegenüber der sich vom streng katholischen Elternhaus unter dem Einfluß eines Schulfreundes emanzipierenden Tochter ${ }^{3^{8}}$, das Nichtakzepticren des Freundes einer erwachsenen Tochter $^{39}$, das Hausverbor für den I'reund der 20jährigen Tochter während der Abwescnheit des verwitweten Vaters ${ }^{4 c}$ oder ein vorausgegangenes mehrjähriges selbständiges Leben eines volljährigen Kindes"1 genannt. Insgesamt bemüht sich die Rechtsprechung, die Bedingungen festzulegen, unter denen ein Zusammenleben noch erwartet werden kann, wobei auch die Autonomiebedürnisse des Kindes bcrücksichtigt werden. Insbesondere fällt in die Waagschale, ob bei einem Zusammenleben mit den Eltern das Studium behindert wird. ${ }^{42}$ Dennoch sind die Kriterien recht

35 BayObLG FamRZ 1986, 930.

36 Siehe dazu auch BayObLG FamRZ 1985, 513 , dem die Literatur wetgehend zustımms.

37 BayObLG NJW 1977,680 .

38 OLG Frankfurt NJW 1977, 1297

39 LG Kiel FamRZ $198_{4}, 193$.

40 OLG Hamburg FamRZ $1989,309$.

4! Vgl. z. B. BayObLG FamRZ 1979, 950, nach funfjahriger Trennung enes volljahrigen Sohnes von den Eltern.

42 OLG Hamburg FamRZ 1987, 1183 
diffus. Seit Beginn der neunziger Jahre gibr es weniger publizierte Entscheidungen, so daß sich dic Vermutung aufdrängt, daß die schwierigere soziale Lage vieler Familien auch von den Kindern stärker mit berüicksichtigt wird.

4. Für das Verhältnis der Generationen zumindest von symbolischer Bedeutung ist ferner die Frage, ob Kinder den Unterhaltsanspruch gegen ihre Eltcrn wcgen unsozialen Verhaltens ganz oder teilweise verlieren können. Maßgeblich dafür ist $\$ r 6 \times 1$ $B G B$, dessen Abs, 2 eine Verwirkung gegenüber minderjährigen unverheirateten Kindern von vornherein ganz ausschließt. Für volljährige Kinder gilt dagegen, daß die Untcrhaltsverpflichtung nach Billigkeit ermäßigt werden oder ganz cntfallen kann, wenn der Unterhaltsberechrigte sich vorsätzlich einer schweren Vcrfehlung gegen den Unterhaltspflichtigen odor einen nahen Angehörigen des Unterhaltspflichtigen schuldig gemacht hat. ": Am meisten diskutiert worden ist hicr der Fall, daß ein volljähriges Kind einen Unterhaltsanspruch geltend macht, zugleich aber jeglichen Kontakt mit dem unterhaltsverpflichteten Elternteil ablehnt. Die überwiegende Auffassung ${ }^{44}$ hält dics nicht für ausreichend, um cine vollständige oder teilweise Verwirkung des Unterhaltsanspruchs anzunehmen. Teilweise wird auch geprüft, ob die Eltern möglicherwcise für den Abbruch der Beziehung allein oder mitverantwortlich sind.

Während in früheren Jahrzehnten zumindest freundliche Postkarten zu Weihnachten und zu Geburtstagen erwartet wurden, zeichnet sich in der Rechtsprechung cine Haltung ab, die den Verzicht auf jegliche Aufmerksamkeiten für maßgeblich erklärt. Auch Taktlosigkeiten werden insoweit ignoriert. Fine schwere Verfchlung, die einc (teilweise) Verwirkung rechtfertigt, setzt vielmehr nach der Rechtsprechung einen schweren Schuldvorwurf voraus. ${ }^{45}$ Danach sollen tiefgreifende Kränkungen, die cinen groben Mangel an verwandtschaftlicher Gesinnung und menschlicher Rücksichtnahme erkennen lassen, zu einer Unterhaltskürzung führen könncn, wic ctwa tätliche Angriftic und Bedrohungen, Denunziationen zur beruflichen oder wirtschaftlichen Schädigung ${ }^{46}$, der unzutreffende Vorwurf sexuellen Mißbrauchs ${ }^{47}$ oder eine tiefgrcifende Verachtung des Unterhaltspflichtigen. ${ }^{4^{8}}$ Die Erklärung eincr Untcrhalt verlangenden Tochter, der tödlich crkrankte Vater existiere als Mensch und Person überhaupt nicht mchr, wird hier als konkretes Beispiel genannt. ${ }^{+9}$ Die Bekundung des bloßen Desinteresses an den Eltern und die völlige Kontaktverweigerung schließen dagegen die langjährige Geltendmachung eines vollen Unterhaltsanspruchs nach der gegenwärtigen Rechtsprechung nicht aus.

Viele Eltern können dies nicht nachvollziehen. Es ist auch schwer zu begreifen, daß eine über mehr als zwej Jahrzehnte hinausgehende Alimenticrung der Kinder nicht einmal zu einer Geburtstagskarte berechrigt. Insowcit ist an dicser Stelle der Abbau des individuellen familiären Generationenaustauschs am pointiertesten spürbar. Wer sich die prakrischen Fallkonstellationen ansieht, wird aber kaum umhin kommen, der Rechtsprechung zu folgen. Was nützt es den Eltern, wenn sie zu besonderen Gelegenheiten formale Zuwendungsborschaften bekommen, aus denen sie auch nur das I'ehlen jeder scelischen Verbindung entnehmen können. Die stereotype Postkarte: "Alles Gute zum Geburtstag wäre letzrlich nur eine Unterwcrfungsgeste, die

43 Die ubrigen Alternativen des $\$ 16 \mathrm{I}$ Abs 1 Satz I BGB fallen demgegenuber nicht ins Gewicht.

44 BGH FamRZ 1991, 322; OLG Munchen FamRZ 1992, 595; OLG Dusseldorf FamRZ 1995, 957; OLG

Celle FamRZ 1993, 1235; a. A. OLG Frankfurt FamRZ 1990, 789 .

45 OLG Frankfurt FamRZ 1991, 1477.

46 OLG Celle FamRZ 1993, 1235.

47 OLG Hamm FamRZ 1995, 958 .

48 OLG Hamm FamRZ $1993,468$.

49 Siehe Palandt-Diederichsen, BGH, 56. Aufh., $\$ 1611$ Rdnr. 3 m.w.N., im Anschluß an Schüz, Anm. zu OLG Bamberg FamRZ 1992, 717, 1338. 


\section{Weitere familienrechtliche Verpflichtungen}

Außcrhalb des Unterhaltsrechts sind Verpflichtungen von älteren Kindern vor allcm in den $\$ \$ 1618$ a und 1619 BGB verankert. Nach $\$ 1618$ a sind Eltern und Kinder einander Beistand und Rücksichtnahme schuldig. Diese zu Beginn der 8oer Jahre ins BGB eingefügte Vorschrift hat den Zweck, Gefährdungen der Familie als Institution entgegenzuwirken. Sie sollte eine Generalklausel für die Familie werden, wie es $\$ 1353$ BGB für die eheliche Lebensgemeinschaft ist..$^{\circ}$ Dabei wird nicht einmal vorausgesctzt, daß Eltern und Kinder noch in derselben Hausgemeinschaft wohncn. Die Norm solltc möglichst cine echte Rechtsverpflichtung bcgründen, hat jedoch seither kaum praktische Bedeutung erlangt. Im Schrifttum wurde vorgeschlagen, wenigstens den Nachhilfeunterricht durch ältere Geschwister, dic Aufrechterhaltung persönlichen Kontakts u. a. unter diese Vorschrift zu subsumieren. Die Rechtsprechung hat aus der Norm etwa hergcleitct, daß ein volljähriges Kind notfalls die clterlichc Wohnung räumen ${ }^{51}$ oder daß die Mutter dem Kind Auskunft über den nichtehelichen Vater geben müsse. ${ }^{52}$ Letzteres ist jedoch außerordentlich umstritten. ${ }^{33}$ Die Auslegung der Vorschrift macht insgesamt deutlich, daß nach dem Wegfall des ökonomischen familiären Generationenaustauschs ein lebendiger sozialer Austausch während des Zusammenlebens nicht durch eine Rechtsnorm gesteuert werden kann. So bleibt es im wesentlichen bei einer Rhetorik der Rücksichtnahme, die ebenso folgenlos zu bleiben scheint wie vielc elterliche Ermahnungen.

Gemäß $₫ 16$ Ig BGB ist das Kind, solangc es dem elterlichen Hausstand angehört und von den Eltern erzogen oder unterhalten wird, verpflichtet, in einer seinen Kräften und seiner Lebensstellung entsprechenden Weise den Eltern in ihrem Hauswesen und Geschäft Dienste zu leisten. Diese sog. familienrechtliche Dienstlcistungspflicht ist ebenfalls ein eher hilfloser Versuch, das donatorische Verhältnis von Eltern zu Kindern mit einem Austauschelement auszustatten. Die Dienstleistungsverpflichtung der Minderjährigen soll von den Eltern mit den Mitteln des elterlichen Sorgerechts durchgesetzt werden. Gegen Volljährige soll sogar eine Leistungsklage möglich sein, ohne daß aus dem erwirkten Urteil jedoch die Vollstreckung soll betrieben wcrden können. ${ }^{54}$

$\$ 1619$ BGB bietet sich zunächst für die Ableitung einer Pflicht zur Mithilfe im Haushalt an. Insbesondere wenn die Eltern berufstätig sund, liegt es nahe, daß sich die minderjährigen Kinder nicht nur bedienen lassen, wobei allerdings ihre schulische Belastung und das Freizeitbedürfnis zu berücksichtigen sind. In den joer Jahren hat der BGH sieben Wochenstunden bei einem 14jährigen Kind für angemessen erklärt."s

Seither hat man in der Judikatur von dieser Verpflichtung jedoch nicht mehr viel gehört. Weitergehende Dienstleistungspflichten können sich ergeben, wenn die Eltern zuhause einer Bcrufstätigkeit nachgehen oder einen Erwerbsbetrieb unterhal-

so BT.Drucks. $8 / 2788$ S. 43 .

s1 AG; Gladbeck FamRZ 1991, 980

s2 AG Gemunden FamRZ 1990, 200.

53 Siche erwa Staudinger/Coester, BGB, 12. Aufl., $\$ 1618$ a Rdnr. 35

$\$ 4$ Entsprechend $\$ 888$ Abs. 2 Z'O

ss BGIINJW 1973. 535 . 
ten. Zu denken ist hierbei insbesondere an den landwirtschaftlichen und handwerk-

lichen Bereich. Aber auch hier steht scit langem fest, daß die Schul- und Berufsausbildung den Vorrang haben muß. Auch sonstige Versclbständigungsinteressen müssen berücksichtigt werden. In wesentlichen wird es heute um die Pflicht zur Mitarbeit im Haushalt gehen, die aber sehr vielc Fltcrn nicht durchzusetzen vermögen. Was die Mitarbeit beim Erwerb der Eltern angeht, so wird juristisch vor allem eine Vergütungspflicht diskutiert. Sie folgt jedoch nicht aus dem Gesetz, sondern muß mit einem Vertragsschluß begründet werden. Ein stillschweigender Vertragsschluß soll sich aus den Umständen ergeben können. ${ }^{36}$

\section{Familienintegration und Kinderpflichten}

Für die Integration des Kindes in die Familie trägt die Rechtsordnung auf vielen Gebieten Sorge. Im Familienrecht ist insbesondere noch die Namensgebung von Bedeutung, die heute keine einfache Angclegenheit mehr ist. Da die Ehegatten nach \$135s Abs. I Satz 3 BGB keinen gemeinsamen Familiennamen (Ehenamen) mehr bestimmen müsscn, bedarf cs nach $\ 1616$ Abs. 2 BGB einer zusätzlichen Bestimmung des Kindesnamens. Nachträglichc Namensänderungen sind in vielfacher Weise möglich. Ab dem s. und dem I 4. Lebensjahr erhält das Kind durch Mitwirkungsrechte einen Zuwachs an sclbständiger Rechtsstellung. Bei der Neuordnung des Namensrechts ist dem Gleichberechtigungsgrundsatz zwischen den Geschlechtern Rechnung getragen worden, wodurch aber die Integrationsleistung des $\mathrm{Na}$ mensrechts reduziert wurde.

Auch außerhalb des Tamilienrechts gibr es eine Vielzahl von Mündigkeitsstufen, mit denen die Verselbständigung des Kindes gegenüber sciner Familie realisiert wird. In einem Lehrbuch des Jugendrechts ${ }^{37}$ hat der Autor versucht, die Altersstufen aller maßgeblichen Gesetze in einer Übersicht zusammenzustellen, von der Mündigkeit hinsichtlich der Religionserziehung bis hin zur Berechrigung, Asylrechtsanträge zu stellen. Aber auch der Experte hat hier die größte Mühe, Vollständigkeit zu crreichen, wenn man alle privatrechtlichen und öffentlichrechtlichen Rechtsgebiete einbezieht. Dabei ergibt sich, daß Jugend in der Bundesrepublik Deutschland eine hochkomplexe regulierte Lebensphasc ist, bci der jedoch wicdcrum zahlreiche Bestimmungen hinter der Lebenswirklichkeit hinterherhinken.

Relativ präzise geregelt sind etwa dic schulrechtlichen Verpflichtungen, ohne daß damit ihre Finhalung in hinreichender Weisc gesichert wäre. Auch hier erweisen sich dic Eltern vielfach als überfordert, die Kinder stetig, pünktlich und aufnahmebereit zur Schule zu schicken oder gar für einen körperlichen und geistigen Zustand zu sorgen, der die Aufnahmefähigkeit und Lernbereitschaft gewährleistet.

Aus den sonstigen Rechtsgebieten sind vor allen noch zwci Fragenkomplexe aufzugreifen. Der eine wird gerade übcr dic Fachöffentlichkeit hinaus diskutiert und betrifft den Beginn der strafrechtlichen Verantwortlichkeit mit der Vollendung des 14. Lebensjahres ( $\$ \times$ Abs. 2 und $\$ 3$ JGG). Aufgrund des früheren Einsetzens der Adoleszenzphase und aufgrund allgemeiner gesellschaftlicher Veränderungen werden Kinder immer früher straffällig. Werden sie nach Aufklärung der Tat einfach nach Hause geschickt, ist die Wiederholungsgefahr beträchtlich und möglicherweise der Einstieg in eine kriminelle Karriere begünstigt. Die Diskussion un eine frühere 
Strafmündigkeit und damit die Möglichkeit von Strafen, insbesondere Freihcitsstrafen für 1 zjährige, ist nicht nur in Deutschland im Gangc. Fine Gesellschaft, die sich hier nur mit Freihcitsstrafen zu helfen wciß, entwickelt wieder barbarische Zügc. Mit der Abwehr von solchen Vorschlägen ist es jedoch nicht getan, da familien- und jugendrechtliche Hilfen als Alternativen entwickelt werden müssen. Bedenkt man den gesellschaftlichen Schaden durch kriminelle Karrieren, dann ist auch ein ins Gewicht fallender finanzieller Einsatz des Staates mittels Familienbetreuern bei krimincll gewordenen Kindern lohnend.

Daß Kinder nicht mit erheblichen finanziellen Verpflichtungen in dic Volljährigkeit entlassen werden dürfen, ist ebenfalls ein noch nicht genügend bearbeitetes Problem. Die deliktsrechrlichen Normen lassen insoweit eine eigenständige Haftung zu, soweit $\operatorname{cin}$ Kind die notwendige Einsichtsfähigkeit hatte. Auf die Stcuerungsfähigkeir wird hier nicht abgestellt. ${ }^{s \AA}$ Es versteht sich, daß größcrc Verbindlichkeiten eine ordnungsgemäße Ausbildung und den Einstieg in den Beruf in gravierender Weise beeinträchcigen können. Neben den deliktsrechtlich.en können auch vorzeitige vertragsrechtliche Verbindlichkeiten begründet werden, sei es, daß die Minderjährigen mit Zustimmung der Eltern bereits Bankverbindlichkeiten eingegangen sind, sei es, daß die Verbindlichkciten aus Geschäften der Eltern im Namen der Kinder stammen. Hier bedarf es der Entwicklung eines konsistenten Konzepts zur Begrenzung der Haftung vor dem Eintritt in die Volljährigkeit.

Ein Lösungsansatz dafür findet sich allerdings nicht in dem mit der Kindschaftsrechtsrcform geplanten Haftungsbegrenzungsgesetz ${ }^{39}$, nach dem Erben und Miterben von Unternehmen und Unternehmensbeteiligungen während einer Dauer von drei Monaren nach Erreichen der Volljährigkeit überprüfen dürfen, ob sie die Haftung aus den ihnen eröffneten unternchmensrechtlichen und gesellschaftsrechtlichen Beteiligungen begrenzen wollen. Bei diesem Gesetzentwurf hat sozialwissenschaftliche Ignoranz Patc gestanden, da es kaum einem jungen Erwachsenen unter dem Druck der Familie möglich sein dürfte, die Risiken einer solchen Beteiligung in hinreichender Weise abzuschätzen.

\section{Schlußfolgerungen}

Kindheit ist in Erwartung der Informationsgesellschaft mit ihrem hohen Qualifikationsniveau, ihrem begrenzten Arbeitskräftebedarf und der Gefahr der AusschlieBung immer breiterer Schichten von der Teilhabe am gesellschaftlichen Rcichtum prekärer geworden als in den vergangenen Jahrzehnten. Der Wegfall des familiären Generationenaustauschs läß̈ sich nicht mittels juristischer Fiktionen neutralisieren, weder mit der Formcl vom Kindeswohl noch mit der ständigen Erweiterung des Katalogs der Kinderrechte ${ }^{60}$ oder einer allgemeinen Postulierung von sanktionslosen Kindespflichren. Die Solidaritätsressourcen der Familie erscheincn hcute als wesentlich geringer als nach dem Zweiten Weltkrieg, wo die Familie praktisch die einzige sozialc Auffanginstitution inmitten einer völlig verunsicherten politischen und sozialen Umwelt war. Der Aufbau ncuer Solidaritätszusammenhänge ist dennoch eine Hoffnung, die nicht aufgegeben werden darf. $\mathrm{Zu}$ unterstützen ist sie auch durch

$58 \mathrm{BGH}$ NJW $1984,1958$.

59 Dadurch soll endlich der Entscheidung des BVerlG vom 13.5. 1986 (BVerfGE 72, 155 ) Rechnung getragen werden.

60 Dagegen unter Bezug auf die franzosısche Diskussıon pointıert Irène Théry, in: Carolıne Steindorff (Hrsg.), Vom Kindeswohl zu den Kindesrechicen, 1994, S. 76. 
wohldurchdachte Einzelreformen, denen jedoch eine hinreichende empirische Erkundung der sozialen Wirklichkeit vorausgehen muß.

Gcrade dies ist bei der Diskussion der Kindschaftsrechtsreform nur ganz unzureichend geschehen. Das Kindeswohl ist teilweise nur als rhetorisches Vchikel verstanden worden, mit dem Frauenrechtc, Männerrechtc und Professionsinteressen besser verfolgt werden können. Die rechtliche Anerkennung einer nicht durch EhcschlieBung begründeten Familie mag dem Kindeswohl dienen, wenn die Eltern trotz ihres Zusammenlebens in keinem Falle zur Heirat bereit sind. Dic Ausblendung der sorgerechtlichen Folgen aus dem Scheidungsprozeß wird ihm gewiß nur schaden. Die gcsellschaftstheoretische Frage, ob und wic im Hinblick auf die schwächer werdenden sozialstaatlichen Ressourcen dic Familie als Solidarinstitution gestärkt werden kann, auch etwa durch ein wcchselseitiges Pflichtgefüge zwischen Lltern und Kindern, ist Icider überhaupt nicht gestellt worden.

Karl-Jürgen Bieback

\title{
Die mittelbare Diskriminierung wegen des Geschlechts
}

\author{
Ihre Grundlagen im Recht der EU und ihre Auswirkungen \\ auf das Sozialrecht der Mitgliedstaaten
}

Nirgendwo ist der Einfluß des Europarechts auf das nationale Arbeits- und Sozialrecht so deulich spürbar geworden wie beim Verbot der miltelbaren Diskriminicrung wegen des Geschlechts. Wie kam es zu dicsem Verbot, welche Ausprägung erfuhr es durch die Rechtsprechung der EuGH, welche wesentlichen Probleme hat es aufgeworfen?

Die vorliegende Studie beantwortet diese Fragen und stellt das Verbot in den Kontext der Verbote der Diskriminierung wegen eines besonderen Merkmals (Geschlecht, Rasse etc.). Bieback untersucht dabei erstmals umfassend und rechtsvergleichend, ob die (zwölf alten) Mitgliedstaaten der EU ihr nationales Recht den gemeinschaftsrechtlichen Vorgaben angepaßt haben. Es zeigt sich, daß zahlreiche Regelungen des Sozialrechts (z.B. zu atypischer Beschäftigung, zur Umschreibung der Risiken und zu den Familienleistungen) gegen das Verbol der mittelbaren Diskriminierung verstoßen.

Das Werk richtet sich gleichermaßen an Wissenschaft und Praxis. Der Verfasser ist Universitätsprofessor für Arbeits- und Sozialrecht an der Hochschule für Wirtschaft und Politik Hamburg und hat zahlreiche Publikationen u.a. zum Sozialrecht und zum Recht der Diskriminierung veröffentlicht.

1997, 25/ S., brosch., 76,-DM, 555,- öS, 69,-sFr; ISBN 3-7890-4957-3 (Schriften zur Gleichstellung der Frau. Bd. 17) 